This item was submitted to Loughborough's Research Repository by the author.

Items in Figshare are protected by copyright, with all rights reserved, unless otherwise indicated.

\title{
Comparison of selective laser melted commercially pure titanium sheet- based triply periodic minimal surfaces and trabecular-like strut-based scaffolds for tissue engineering
}

\section{PLEASE CITE THE PUBLISHED VERSION}

https://doi.org/10.1002/adem.202100527

\section{PUBLISHER}

Wiley

\section{VERSION}

VoR (Version of Record)

\section{PUBLISHER STATEMENT}

This is an Open Access Article. It is published by Wiley under the Creative Commons Attribution 4.0 Unported Licence (CC BY). Full details of this licence are available at: http://creativecommons.org/licenses/by/4.0/

\section{LICENCE}

CC BY 4.0

\section{REPOSITORY RECORD}

Torres, Carmen, James Borgman, Ben Sargeant, Hugo Bell, Enrique Alabort, Craig Lindsay, and Paul Conway. 2021. "Comparison of Selective Laser Melted Commercially Pure Titanium Sheet-based Triply Periodic Minimal Surfaces and Trabecular-like Strut-based Scaffolds for Tissue Engineering". Loughborough University. https://hdl.handle.net/2134/16627366.v1. 


\title{
Comparison of Selective Laser Melted Commercially Pure Titanium Sheet-Based Triply Periodic Minimal Surfaces and Trabecular-Like Strut-Based Scaffolds for Tissue Engineering
}

\author{
Carmen Torres-Sanchez,* James M. Borgman, Ben Sargeant, Hugo Bell, \\ Enrique Alabort, Craig Lindsay, and Paul P. Conway
}

This systematic comparison between sheet-based triply periodic minimal surfaces (TPMS) and strut-based ordered and disordered lattice topologies offers insights into parametric designs for tissue engineering scaffolds intended as implants. The study explores the effect of topology on compressive properties and in vitro osteoblastogenesis. TPMS-sheet gyroid and IWP, Voronoi tessellation with different sharpness, and cubic orthogonal lattices are studied. Disparities between the design intent and the as-manufactured scaffolds, intrinsic to selective laser processing, are considered to ensure that actual porosity and surface area per unit volume, two important factors in tissue engineering, are consistent across the set. Surface analysis reports the presence of microporosity created by partly sintered titanium particles. The TPMS topologies display a stretching-dominated deformation and the strut-based disordered ones a bending-dominated double-shear failure. Although the trabecular-like structures exhibit an enhanced compressive behavior when the designed topology is smoothed, they are more prone to printing imperfections with sharper finishes. The in vitro studies reveal that the trabecular sharp topology displays a faster proliferation rate, explained by concavity-driven cellular growth, but its smooth counterpart promotes a larger differentiation extent, outperforming TPMS, as it is aided by larger pore throats lined with microporosity at the scale of osteoblastic geometric features.

\section{Introduction}

An opportunity is offered by porous structures as implants in medical applications and arthroplasties because their mechanical and physical properties can be tuned to match patient-specific needs. For ex vivo research, there is merit in using 3D models instead of 2D layers in tissue engineering to recapitulate cell growth in microenvironments that are similar to native tissue, because this narrows the gulf between in vitro tests and clinical translation. ${ }^{[1]}$ Titanium and its alloys have been researched extensively because of their biocompatibility, high strength, low wear, and corrosion resistance (due to the passivating oxide layer spontaneously formed on the surface $\left.{ }^{[2]}\right)$. When embodied as porous structures, they offer mechanical properties that can match those of cortical and trabecular bone, ${ }^{[3]}$ avoiding stress shielding and biomechanical failure. Porous scaffolds must be conducive to osseointegration by means of providing channels and interconnected pores for nutrient distribution, ${ }^{[4]}$ networks for cellular proliferation, differentiation and maturation, and ultimately for bone healing. ${ }^{[5]}$

Much work has explored different pore architectures and sizes and routes by which they can be physically realized. Advances in computer-aided design (CAD), multiphysics modeling, ${ }^{[6]}$ and additive manufacturing (AM) technology have allowed the definition of a design space to digitally test manufacturability boundaries for porous structures with the desired physical, mechanical, and permeability properties that can enhance biological behavior. ${ }^{[7]}$ Techniques such as selective laser melting (SLM) or electron beam melting (EBM) have been demonstrated as practical processing routes to achieve both parametric and nonparametric designs, ordered or random, using metals. With regard to parametric designs, triply periodic minimal surface (TPMS) structures ${ }^{[8]}$ have received attention in recent years as AM managed to realize the manufacture of these topologies that offer mechanical superiority due to a uniformly distributed load transfer, free of discontinuities and self-intersecting elements, and 
therefore less prone to printing imperfections. Research to date has explored TPMS as a promising topology to emulate bone function. Parametric designs can also generate (pseudo-)random structures, for example, trabecular like. These structures remain attractive designs because they are based on the design principle that function follows form. It is accepted that there is a lack of systematic comparison that comprehensively includes the design of the porous structures and both their mechanical and biological performance, derived from that topology design. ${ }^{[9]}$

The ultimate design intent for a structure to be successful in tissue engineering applications needs to accommodate a set of "hygiene factors" that include a noncytotoxic material; a pore size larger than $300 \mu \mathrm{m},{ }^{[4]}$ more typically larger than $600 \mu \mathrm{m}$ for in vivo applications, ${ }^{[10]}$ to promote osteoconduction; and porosity larger than $50 \%,{ }^{[4]}$ typically larger than $60 \%$ in commercial trabecular metal products ${ }^{[\mathrm{a}]}$ to promote permeability as well as maintain mechanical integrity to sustain physiological loads, especially for load-bearing applications. Another important property of the tissue engineering embodiment is a high surfaceto-volume ratio available for scaffold-cell interaction and implantbone fixation. ${ }^{[5]}$ Little attention has been given to the effect of pore morphology independent of surface area. Many studies have dealt with changes in pore size and/or porosity, while keeping one of them constant; however, surface area would vary as a consequence. ${ }^{[10,11]}$ A systematic study where surface area is kept constant and pore morphology varied would help elucidate the true effect of pore size and porosity on cellular behavior. Therefore, the coupled consideration of macroporosity (defined by the pore geometry and topology) and microporosity (by the small pores and wall roughness $)^{[1 a, 5]}$ is relevant and important.

This present study provides a systematic comparison between parametric designs (two sheet-based TPMS topologies gyroid and IWP, selected for their high permeability ${ }^{[12]}$ ) and a Voronoi tessellation with two degrees of smoothening, chosen for biomimicry, ${ }^{[13]}$ as well as a nonparametric orthogonal bodycentred cubic lattice to establish the true contribution of pore topology to mechanical and in vitro performance. Unlike other studies, the design intent focused on porosity and surface area, rather than on pore size. The scaffolds were manufactured by SLM using commercially pure titanium (cpTi) and their physical, geometrical, compressive, and osteoconductive properties were evaluated. Deviations between the design intent and the as-manufactured scaffolds, arising from the printing technique, created microporosity and disparities in physical and geometrical features. Topology and microporosity were both considered to

(a)

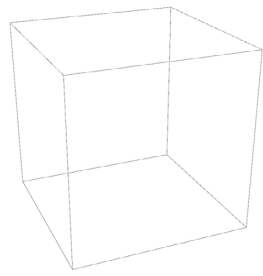

(b)

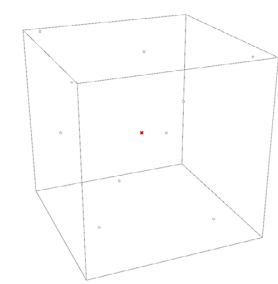

(c)

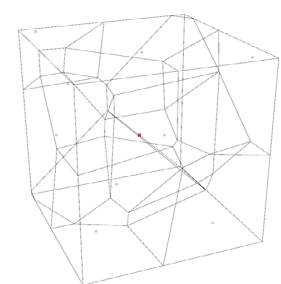

elucidate their effect on both mechanical performance and osteoblastogenesis over a long-term study of 28 days.

\section{Experimental Section}

\subsection{Design of the scaffold structure}

The selected structures were two sheet-based TPMS (gyroid and IWP) and two strut-based trabecular (sharp and smooth) types. Preliminary studies considered other designs (e.g., primitive and diamond) but it was not technically possible to warrant the desired levels of open porosity and percolation due to pore occlusion in those designs. For comparative purposes, particularly in relation to mechanical and biological performance, a strut-based cubic lattice was also designed. The intended porosity was $75 \%$ with an intended surface area per unit volume in the range $30-40 \mathrm{~cm}^{-1}$. As porosity and surface area were the two parameters intended to be consistent, the pore and strut sizes were varied depending on the unit cell design. The TPMS structures were generated in Grasshopper (Rhino 6.0., USA) using Equation (1) and (2) without further topology optimization.

Gyroid

$\cos (x) \sin (y)+\cos (y) \sin (z)+\cos (z) \sin (x)=0$

IWP

$$
\begin{gathered}
2[\cos (x) \cos (\gamma)+\cos (\gamma) \cos (z)+\cos (z) \cos (x)] \\
-[\cos (2 x)+\cos (2 z)+\cos (2 \gamma)]=0
\end{gathered}
$$

These structures were generated from designated unit cells. The surfaces were thickened in Magics v.22.03 (Materialise, Belgium) to achieve the intended porosity and surface area. The cubic lattice was generated by repeating a $1.4 \mathrm{~mm}$ "cross" with $0.81 \mathrm{~mm}$ overlap, that created the unit cell.

The trabecular structures used the Voronoi tessellation method of the Rhino software package (Figure 1). First, a cube region, whose outer surfaces defined the overall volume of the porous scaffold, was generated. A random cloud of points was distributed within that volume. These points acted as seeds to create a 3D Voronoi tessellation within that volume. The edges of the Voronoi structure were then used to define a series of surfaces that formed the porous structure. To generate polyhedron cells, separated from each other at a distance proportional to the Voronoi cell structure, the 3D Voronoi structure was broken into

Figure 1. Design of the strut-based trabecular model: a) bounding volume; b) random point generation ( $n=10$ in this example); c) Voronoi tessellation; d) surface generation; e) volume generation to produce a "sharp" design; and f) Catmull-Clark smoothening to generate a "smooth" design. 
points, edges, and faces to allow an equidistant copy of these at an offset, which controlled the strut thickness and thus the relative density of the lattice structure. Finally, the volume within the surfaces was "filled" to create the solid trabecular structure, which generated the strut-based trabecular "sharp" (T-sharp) porous structure. An additional step was used to generate the trabecular "smooth" (T-smooth) structure using the Catmull-Clark algorithm subdivision smoothening step built into Rhino's "Weaverbird." The number of the nucleating points (or seeds) was controlled such that the lattice surface area-to-volume ratio could be modified for constant porosity. No topology optimization was conducted.

\subsection{Manufacture of the Scaffolds}

The scaffolds were prepared using cpTi grade 1 powder in an SLM AM process. The spherical gas-atomized powder (AP\&C, GE Additive, Canada) displayed a distribution in the range $45-15 \mu \mathrm{m}$ of D10 $(19 \mu \mathrm{m}), \mathrm{D} 50(34 \mu \mathrm{m})$, and D90 $(47 \mu \mathrm{m})$, as per testing method ASTM B822-17; a chemical composition: (wt\%) C (0.01), O (0.10), N (0.01), H (0.002), Fe (0.03), other $(<0.2)$, as per ASTM E1941-10, E1409-13, E1447-09, E2371-13, and density $2.63 \mathrm{~g} \mathrm{~cm}^{-3}$, as per ASTM B212-17.

The SLM process was conducted in a TruPrint 1000 LMF (Trumpf, Germany) equipped with a $200 \mathrm{~W}$ Nd:YAG solid-state laser of $55 \mu \mathrm{m}$ spot size under argon atmosphere ( $<100$ ppm oxygen content). The preliminary study that established the printing parameters can be found in Supporting Information. The laser scanning parameters used were laser beam power of $140 \mathrm{~W}$ at a speed of $1,414 \mathrm{~mm} \mathrm{~s}^{-1}$, hatch distance of $0.11 \mathrm{~mm}$, and a layer thickness of $0.03 \mathrm{~mm}$ to achieve volumetric energy density of $30 \mathrm{~J} \mathrm{~mm}^{-3}$. A zigzag scanning strategy with $90^{\circ}$ rotation between the layers, with a border offset of $0.05 \mathrm{~mm}$, was utilized. There were three sets of printed scaffolds on titanium build plates to suit the subsequent measurements and characterization methods: set 1 , used for mechanical testing and $\mu \mathrm{CT}$ reconstruction, cubes of side $10 \mathrm{~mm}$, including a subset with $2 \mathrm{~mm}$ top/bottom solid layers, as per BS ISO 13314; set 2, used for wall roughness measurements via mercury injections, cylinders of $10 \mathrm{~mm}$ diameter and $10 \mathrm{~mm}$ height; and set 3 , for biological studies, short cubes of $10 \times 10 \mathrm{~mm}$ base and $2 \mathrm{~mm}$ height. The scaffolds were excised (IsoMet High Speed Saw, Buehler, Germany) and ground (AutoMet 250, Buehler, Germany) using SiC paper of 180 and 240 grit for $10-15 \mathrm{~s}$ to achieve parallel planes. The scaffolds were then ultrasonically cleaned in a sequential routine of soapy water $(2 \mathrm{~h})$, acetone $(1.5 \mathrm{~h})$, and 2 -isopropanol $(1.5 \mathrm{~h})$ (Alfa Aesar, UK) to remove impurities and loose particles.

\subsection{Measurements of Models, Physical Characterization, and Volumetric Measurements}

Volume $\left(V_{\mathrm{L}}\right)$, surface area, pore size, wall thickness, or strut size of the CAD models were measured using Blender v2.92. The porosity in the as-designed models was calculated by the ratio $V_{\mathrm{L}} / V$, where $V_{\mathrm{L}}$ is the volume of the porous scaffold and $V$ is total volume. The total porosity of the as-manufactured scaffolds was measured using $(1-D), D$ being the density calculated as the ratio mass divided by volume, that is, "dry porosity."
The open porosity was measured using Archimedes' method with acetone, a low-surface tension liquid, as the immersion medium. Cubes $(10 \times 10 \times 10 \mathrm{~mm})$ were scanned in a micro computed tomography $(\mu-\mathrm{CT})$ system (XT H 160 Xi-Tek, Nikon Metrology, UK) with $8.64 \mu \mathrm{m}$ resolution at $100 \mathrm{kV}$ and $90 \mu \mathrm{A}$. The acquisition was obtained by angular rotation of $0.12^{\circ}$ and without beam hardening. 3015 2D images were collected for each specimen and the volumetric reconstruction was achieved in VG Studio Max 3.1 (Volume Graphics GmbH, Germany). The volumes were exported, segmented, and reconstructed in Fiji ${ }^{[14]}$ to generate an STL file. The image stack was binarized, thresholded, and resampled at factor 4 . These parameters were optimized for consistency among the sets and to ensure features of wall roughness were not lost from the original $\mu \mathrm{CT}$ scan resolution. The pore size and wall thickness or strut size were measured in Fiji using 2D stacks. The volumetric representations were used for surface area and contour size (i.e., mesh size) measurements in Blender (Blender v2.92). Gaussian local curvature on both the models and reconstructed volumes was measured in MatLab (R2019a, Mathworks, UK) analyzed with "pathcurvature" function.

Observation of scaffold cross sections was done by field emission scanning electron microscopy (FESEM JSM $7800 \mathrm{~F}$, JEOL, Japan) at an accelerating voltage of $5 \mathrm{kV}$. To assess microporosity on the walls of the manufactured scaffolds, mercury injection was conducted using an Autopore-IV 9500 porosimeter (Micromeritics Instrument Corp, USA) to determine pore throat size distribution and generate specific cumulative pore area as a function of pore throat size to give an indication of the total available surface area to the preosteoblasts. Mercury was injected into the sample at incremental pressure steps from 1.5 to 500 psia using air and then hydraulic oil as the displacing medium until equilibrium (i.e., the rate of intrusion dropped below $0.001 \mu \mathrm{Lg}^{-1} \mathrm{~s}^{-1}$ ), which was equivalent to $1 \mu \mathrm{m}$ pore throat size when all injection ceased.

\subsection{Mechanical Characterization}

Quasistatic compression testing was conducted as per BS ISO 13314 standard using a UTM (Instron 3369, UK) with a $50 \mathrm{kN}$ (Instron 2530-445, UK) load cell. A constant strain rate of $0.1 \% \mathrm{~s}^{-1}$ was applied, and the displacement of the top platen was measured with an linear variable differential transformer (LVDT) (Instron 2601-062, UK) to reduce compliance errors. Samples were loaded in monotonic compression and increasing strain tests were conducted for validation. Recordings of the compression were captured in 1080 P HD using a Nikon D850 camera and digital image correlation (DIC) measurements were carried out using an open-source 2D digital image correlation for MatLAB (Ncorr v1.2). Strain was calculated as the LVDT displacement divided by the original specimen's height. Stiffness was calculated from the linear slope displayed in the elastic region of the stress-strain curves. Compression strength was calculated using the $0.2 \%$ strain offset method.

\subsection{Biological Performance}

The scaffolds were sterilized twice in an autoclave at $121^{\circ} \mathrm{C}$ for $1 \mathrm{~h}$ followed by thorough rinsing with deionized sterile water. 
The scaffolds were preconditioned by soaking in culture media overnight. MC3T3-E1 murine preosteoblasts were cultured in standard flasks (ThermoFisher, UK) with minimum essential media (alpha-MEM) $\alpha$-MEM (Gibco, UK), supplemented with $10 \%$ fetal bovine serum (FBS) (Gibco, UK), 1\% L-glutamine (Gibco, UK), and 1\% penicillin-streptomycin (Gibco, UK). Preosteoblasts were incubated at $5 \% \mathrm{CO}_{2}$ and $37^{\circ} \mathrm{C}$ (Thermo Scientific Heracell 150, UK) and used at passage 15 . After reaching 70-80\% confluence, preosteoblasts were detached using trypsin-EDTA $(0.25 \% \mathrm{w} / \mathrm{v}$ trypsin/0.2\% EDTA, Gibco) and the cellular solution was centrifuged $(150 \times \mathrm{g}, 5 \mathrm{~min})$. After preliminary studies to optimize 3D seeding efficiency (Supporting information), $1.8 \times 10^{5}$ preosteoblasts were seeded onto the preconditioned scaffolds in low-adhesion 24-multiwell plates (Corning Costar, UK). The media was topped up to $1 \mathrm{~mL}$. Cellular culture medium was replaced every 2 days for the duration of the experiment.

Cellular attachment and proliferation were monitored using a viability reagent (PrestoBlue, Invitrogen, ThermoFisher Scientific, UK) over a 13-day period. This assay utilized a blue, nonfluorescent, cell-permeable, nontoxic, and resazurin-based compound, that upon entering live cells was reduced to resorufin, a red compound that was highly fluorescent. Due to its light sensitivity and to prevent overexposure of cells to PrestoBlue reagent, two plates were used in parallel, to maximize the number of consecutive time points and ensure robustness in the results. Cellular attachment was quantified as per the preosteoblasts attached to the scaffolds in the first $24 \mathrm{~h}$ of culture. The remaining 12 days in the period were used to determine the metabolic activity and determine the phenotypical switch point from proliferation to differentiation of this cell line within these scaffolds. As per the manufacturer's protocol, $500 \mu \mathrm{L}$ of PrestoBlue media were added to each sample and left to incubate in a humidified environment for $90 \mathrm{~min}$ in the absence of light. $150 \mu \mathrm{L}$ of media were then transferred to a black, clear-bottom 96-well plate (Corning, UK) for fluorescence analysis, using a spectrophotometer (Fluostar Omega, UK) (Ex:560/Em:590). A cell standard line was used to transform fluorescence values into cell numbers. Cellular observation was done at $24 \mathrm{~h}$ from seeding and by fixation of the preosteoblasts $(1 \mathrm{~h}$ immersion in 4\% Paraformaldehyde [Sigma-Aldrich, UK]). These were then stained by immersion in a 1:1000 Phalloidin (Invitrogen, ThermoFisher, UK)-PBS (Gibco, UK) solution for $1 \mathrm{~h}$ followed by immersion in 1:2500 (4',6-diamidino-2-phenylindole, DAPI (ThermoFisher, UK)-PBS solution. The preosteoblasts were observed with fluorescent microscopy (Nikon Ti Eclipse, Japan). Cellular interactions with microporosity were observed using electron microscopy (as above) after fixating the cells using the procedure reported in the study by TorresSanchez et al. ${ }^{[15]}$

Cellular volume and extent of differentiation were quantified by means of DNA, protein content, and alkaline phosphatase (ALP) level measurement on the cell lysates. A cell lysate was obtained by removing samples from culture at predetermined time points (day 14, 21, and 28), washing them with PBS (Gibco, UK) three times, and submerging them in $500 \mu \mathrm{L}$ of 0.1\% Triton-X (Sigma-Aldrich, UK) diluted in DNA-free water (Fisher Scientific, UK). These samples were stored at $-80^{\circ} \mathrm{C}$ prior to analysis. A quintuplicate of freeze-thaw cycles was conducted to ensure proper cell lysis, after which the cell lysate was collected and transferred to a $1.5 \mathrm{~mL}$ Eppendorf. DNA content was quantified using the Quanti-iT PicoGreen dsDNA Assay Kit (Thermofisher, UK). This applied an ultrasensitive fluorescent nucleic acid stain to bind to double-stranded DNA (dsDNA), forming a highly fluorescent complex to quantify as little as $25 \mathrm{pg} \mathrm{mL}^{-1}$ of dsDNA. $25 \mu \mathrm{L}$ of each cell lysate solution were mixed with $75 \mu \mathrm{L}$ of $1 \mathrm{X}$ Tris-EDTA buffer solution in a black, clear-bottom 96-well plate. $100 \mu \mathrm{L}$ of PicoGreen solution (prepared as per the manufacturer's protocol) were added to each sample well and left to incubate (at room temperature) for $5 \mathrm{~min}$ in the absence of light, after which fluorescence analysis was completed using a spectrophotometer (Ex:480/Em:520). Fluorescence values obtained were then quantified into DNA content, via the use of a previously prepared DNA standard curve, following the manufacturer's protocol.

The protein content of preosteoblasts, indicative of cellular volume, was quantified using the Pierce $660 \mathrm{~nm}$ reagent (Sigma Aldrich, UK). This method utilized the binding of a metallic dye complex (brown in color) to proteins that resulted in deprotonation of the dye that subsequently shifted color to green in acidic conditions. $10 \mu \mathrm{L}$ of cell lysate were pipetted into a black, clearbottom 96-well plate, after which $150 \mu \mathrm{L}$ of Pierce reagent were added, left to incubate for $5 \mathrm{~min}$, and the absorbance reading was obtained $(660 \mathrm{~nm})$. Protein content was normalized by the DNA content and was therefore relative to the protein content per cell on each sample.

ALP content, a key indicator of osteoinduction, was quantified using the 4-MUP reaction. 4-MUP is a fluorescent substrate for ALP and turns blue once phosphorylated. This reaction can be halted by the addition of Tris-EDTA buffer solution. $50 \mu \mathrm{L}$ of cell lysate were pipetted into a black, clear-bottom 96-well plate, diluted by $50 \mu \mathrm{L}$ of $\mathrm{dH}_{2} 0$, and followed finally by the addition of $50 \mu \mathrm{L}$ of 4-MUP solution; this was left to incubate (at room temperature) for $30 \mathrm{~min}$. $50 \mu \mathrm{L}$ of $100 \mathrm{mM}$ Tris-EDTA (SigmaAldrich) were then added to stop the reaction. Finally, florescence was measured using a spectrophotometer (Ex:360/Em:440). ALP content was normalized by the DNA content and was therefore reported as the relative ALP content the ALP content per cell on each sample.

\subsection{Statistical Analysis}

Measurements were completed in quadruplicate $(n=4)$ unless otherwise stated. Statistical analysis was conducted using GraphPad Prism (v9.1). Statistical significance in the comparison of as-designed and as-manufactured scaffolds was conducted using a two-tailed $t$-test. Normality of data distribution in the biological results was confirmed using the Shapiro-Wilk normality test and any significant difference in data was detected using a one-way analysis of variance (ANOVA) with Fisher's least significance difference (LSD) multiple-comparison posthoc test. When data did not conform to normal distribution, significance was instead determined using a Kruskal-Wallis test, with Dunn's multiple-comparison posthoc test. The threshold for statistical significance was set at $p<0.05$. 


\section{Results and Discussion}

\subsection{Design of Scaffolds}

The study intent was to control surface area within a range across all topologies for comparative purposes. Because this depends on porosity, the design work focused on the effect unit cell number had on surface area when the as-designed porosity was kept constant. As it is well known that the actual porosity of SLM scaffolds is lower than the as-designed porosity, ${ }^{[12,16]}$ an iterative study to quantify the decrease in actual porosity on the as-printed scaffolds was conducted (Figure 2). For this purpose, preliminary specimens in the five designs were prepared and measured in CAD (as-designed, solid squares in Figure 2) and prediction lines were drawn to locate the interpolated unit cell number that would yield the desired surface area per volume ratio. The specimens were then manufactured and their "dry" porosity measured (as-manufactured actual porosity, blank squares in Figure 2), which allowed a prediction of the surface area-to-volume ratio value, accounting for the effects of SLM manufacturing. These preliminary studies informed that the manufactured T-sharp set would incur a further decrease in surface area; hence, the initial designed porosity was slightly higher than the rest (Figure 2d). It was determined that the as-designed TPMSgyroid structure required 216 unit cells, TPMS-IWP 125 unit cells, T-smooth and T-sharp 833 unit cells, and cubic lattice 1728 unit cells.

\subsection{Characterization of Models and Scaffolds}

The measurements of porosity, pore size, strut size, surface area (reported as the surface area-to-total volume ratio) and wall roughness (measured on the reconstructed volumes from $\mu \mathrm{CT})$ on the as-designed CAD models and the as-manufactured printed scaffolds are shown in Table 1. Results obtained from Gaussian curvature can be found in Supporting information. A visual comparison between the as-designed and as-manufactured and reconstructed scaffolds is shown in Figure 3 . The disparities presented in the porosity indicate that the target porosity was not achieved with the SLM printed scaffolds, despite the compensation efforts described in Section 3.1. This was expected as it has been widely reported in literature. ${ }^{[12,13,16]}$ While the Archimedes' method (using acetone as immersion medium) has been reported to yield very accurate results, ${ }^{[17]}$ the departure of the actual porosity from the design intent is clear. The pore sizes were smaller in the as-manufactured TPMS samples, resulting in a decrease in actual porosity. The wall thickness was wider in the sheet-based TPMS scaffolds but the strut size narrower in the strut-based scaffolds (i.e., T-smooth, T-sharp, and the cubic lattice). This narrowing is thought to be related to the laser beam creating a thinner strut because the offset parameter, intended to ensure that the edge of the melt pool matches the CAD geometry, was over compensated and thereby created thinner material beams, in particular, the struts parallel to the build direction. The widening of the struts or sheets has been also reported in other studies and is due to parasitic mass at the curb struts or corners and overmelting of the particles ${ }^{[18]}$ and differences in heat transport between the powder and solid material, leading to the loose powder sintered to the surfaces. ${ }^{[16]}$ It has also been observed that the extra materials are mostly distributed in places perpendicular to the building orientation, ${ }^{[19]}$ and this was also seen in the printed samples (esp. cubic lattice scaffolds in Figure 3e, front row). There were no statistical differences between as-designed and asmanufactured pore sizes (Table 1), indicating that the discrepancies are independent of topology design and intrinsic to the manufacturing process. However, there is statistical significance in the gyroid's and cubic Lattice's strut (or wall) thickness, strongest in the latter, indicating that the deposition of the extra materials is more prevalent in that topology design, supporting the statement earlier.

\subsubsection{Surface Area and Wall Roughness}

The surface area (normalized by volume, $\mathrm{cm}^{-1}$ ) measured on the as-manufactured scaffolds was larger than the design intent, despite attempts made to predict it, as explained in Section 3.1. A decrease in porosity (actual vs design) derives a decrease in surface area for the TPMS and an increase for the trabecular-like ones, as shown in Figure 2. A decrease in unit cell number decreases the surface area in cubic lattice structures. However, the increase in surface area in the as-manufactured scaffolds was of a larger magnitude than expected (see Table 1, surface area/volume). A fractal effect emanating from the wall roughness produced by the presence of partly sintered particles is thought to be a major contributor to this. The presence of satellite and (partly) melted cpTi particles (known as "secondary roughness" ${ }^{20]}$ and widely reported in porous structures processed by SLM ${ }^{[16 a, 21]}$ ) on the walls and struts of all the structures (Figure $4 \mathrm{a}-\mathrm{c}$ ) created a microscale roughness, contributing to the (a)

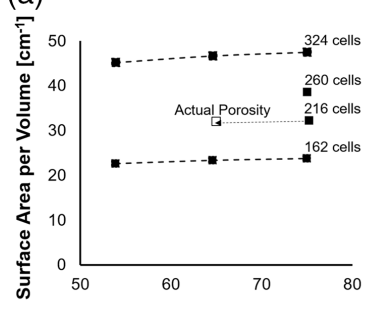

(b)

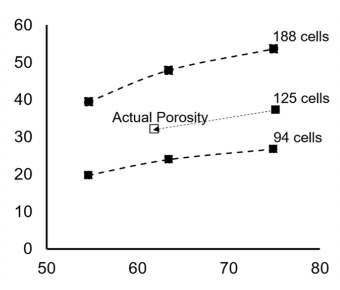

(c)

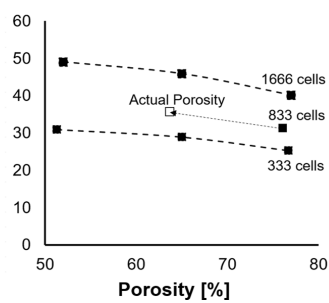

(d)

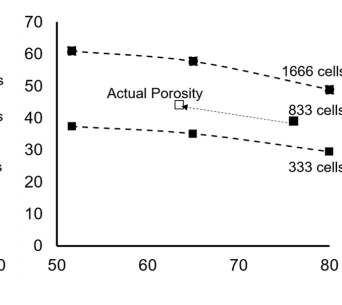

(e)

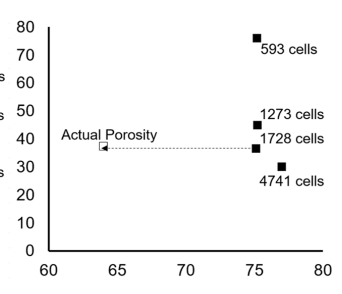

Figure 2. Surface area-per-volume values as a function of porosity for as-designed (black squares) and as-manufactured (blank squares) scaffolds. a) TPMS-Gyroid, b) TPMS-IWP, c) T-smooth, d) T-sharp, and e) cubic lattice, allowing prediction of decrease in actual porosity in the as-manufactured scaffolds. This tool allows the selection of unit cell number in the initial CAD designs. 
decrease in actual porosity (compared with the as-designed porosity) (Figure $4 \mathrm{f}-\mathrm{j}$ ). Corner and strut-joint occlusion was particularly marked in strut-based T-sharp and cubic lattice scaffolds (Figure $4 \mathrm{i}, \mathrm{j})$. This secondary roughness did not deter preosteoblastic attachment (Figure 4d,e). There were no statistical differences in the roughness of the reconstructed as-manufactured among the different topologies (Table 1), adding to the premise that secondary roughness arises independently from topology design and it is intrinsic to the SLM process.

The contour size (i.e., roughness) values measured on the reconstructed $\mu \mathrm{CT}$ models of the scaffolds sits in the bracket size $45-50 \mathrm{~mm}$ (Table 1), which matches the mean particle size of the raw powdered material and is lower than the pore throat radius (Figure 5, inset). This result further confirms the appropriate selection of the resampling factor at the time of volumetric reconstruction. As this roughness has been reported to impact mechanical performance, especially as crack initiation sites, ${ }^{[22]}$ and surface roughness is a well-known factor driving osteoblastic behavior, ${ }^{[23]}$ further studies on microporosity created by the satellite and partly sintered particles were conducted. Figure 5 shows a size distribution of the throat (or wells) radii created by these sintered particles and the cumulative pore area (per g) of these with respect to the pore throat radii. For pores with a radius $10 \mu \mathrm{m}$ and larger, there are only small differences between the designs, and there is a positive correlation with porosity, that is, larger actual (acetone) porosity yields larger cumulative pore areas. Those pores are desirable for essential osteoconduction and vascular tissue ingrowth. There is a switch at $7 \mu \mathrm{m}$, and at $<6 \mu \mathrm{m}$ the specific cumulative pore area values diverge. When $1 \mu \mathrm{m}$ pore throat radii are achieved, the total cumulative pore area increases markedly in the TPMS designs (IWP: $108.64 \mathrm{~cm}^{2} \mathrm{~g}^{-1}$ and gyroid: $102.32 \mathrm{~cm}^{2} \mathrm{~g}^{-1}$ total) when compared with the trabecular designs (T-smooth: $78 \mathrm{~cm}^{2} \mathrm{~g}^{-1}$ and T-sharp: $71 \mathrm{~cm}^{2} \mathrm{~g}^{-1}$ ). These small pores, that is, microporosity, attributed to the wall's microroughness, contribute more to the specific cumulative pore area in TPMS scaffolds when the pore throat radius is $<7 \mu \mathrm{m}$. The cumulative pore area increases sharply for IWP, indicating that there are many pores of very small sizes, probably in the IWP "neck" area of the design, as depicted in the first peak of pore throat radius distribution (Figure 5, inset). On the contrary, the cumulative pore area increases gradually in the T-sharp, suggesting that there are fewer very small pores (Figure 5, inset, $<4 \mu \mathrm{m}$ ). Although the cumulative pore area is shown in Figure 5, spanning two orders of magnitude for the radii, it is plausible to restrict the true area available to preosteoblasts using a high and a low boundary. Preosteoblasts have been found to bridge gaps only when these are shorter than $200 \mu \mathrm{m}^{[24]}$ (i.e., equivalent to $100 \mu \mathrm{m}$ throat radii). While the definition of a low boundary cannot be determined accurately because the preosteoblasts cannot be viewed in situ in this study, throat radii of $\approx 5 \mu \mathrm{m}$ is an informed estimation as $10-15 \mu-m$ is the typical size of preosteoblast dendrites and pseudopodia, used by the cells for attachment and mobility (Figure $4 \mathrm{e}$ and as observed in other studies ${ }^{[5,11,25]}$ ). The shaded area in Figure 5 draws attention to the larger cumulative pore area at that scale in T-smooth scaffolds, presenting an advantage over the other structures and also displaying wider throats (Figure 5, inset). Increased specific surface area at micrometer 


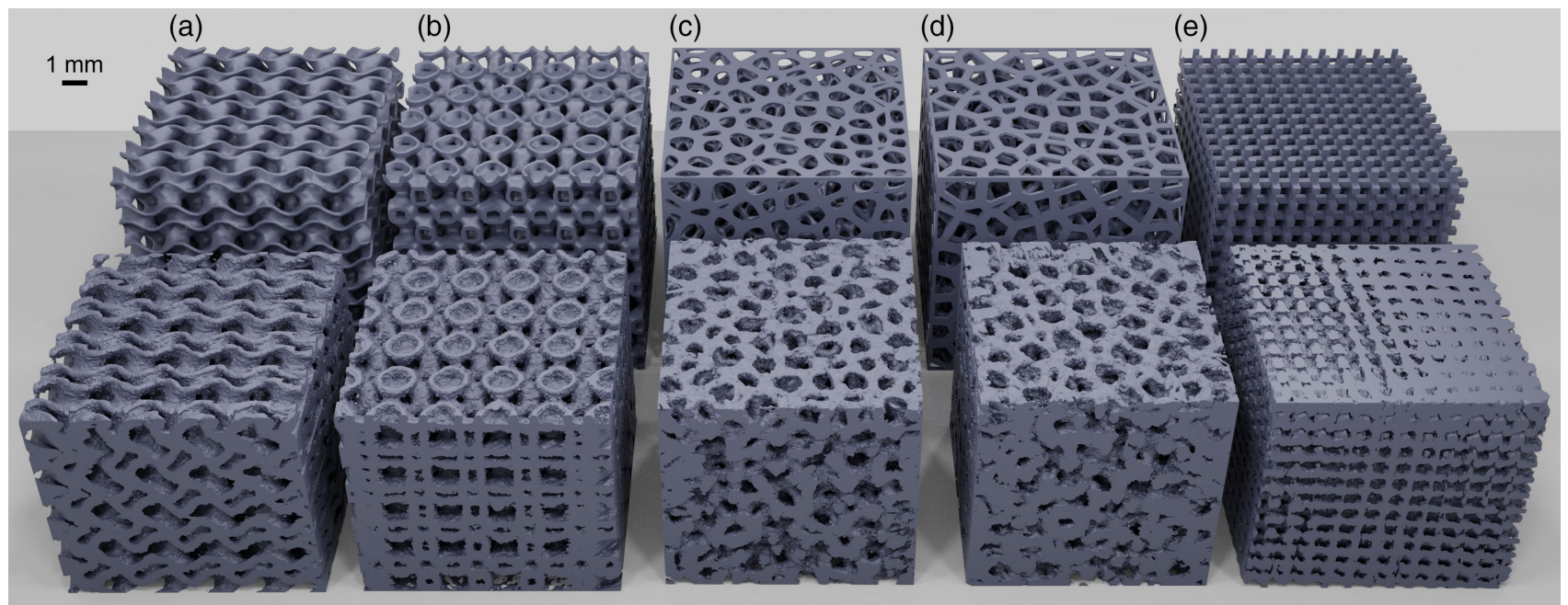

Figure 3. a) TPMS-Gyroid, b) TPMS-IWP, c) T-smooth, d) T-sharp, and e) cubic lattice (back row) CAD designs, and (front row) printed and reconstructed scaffolds depicting surface roughness and imperfections incurred in the manufacturing process.
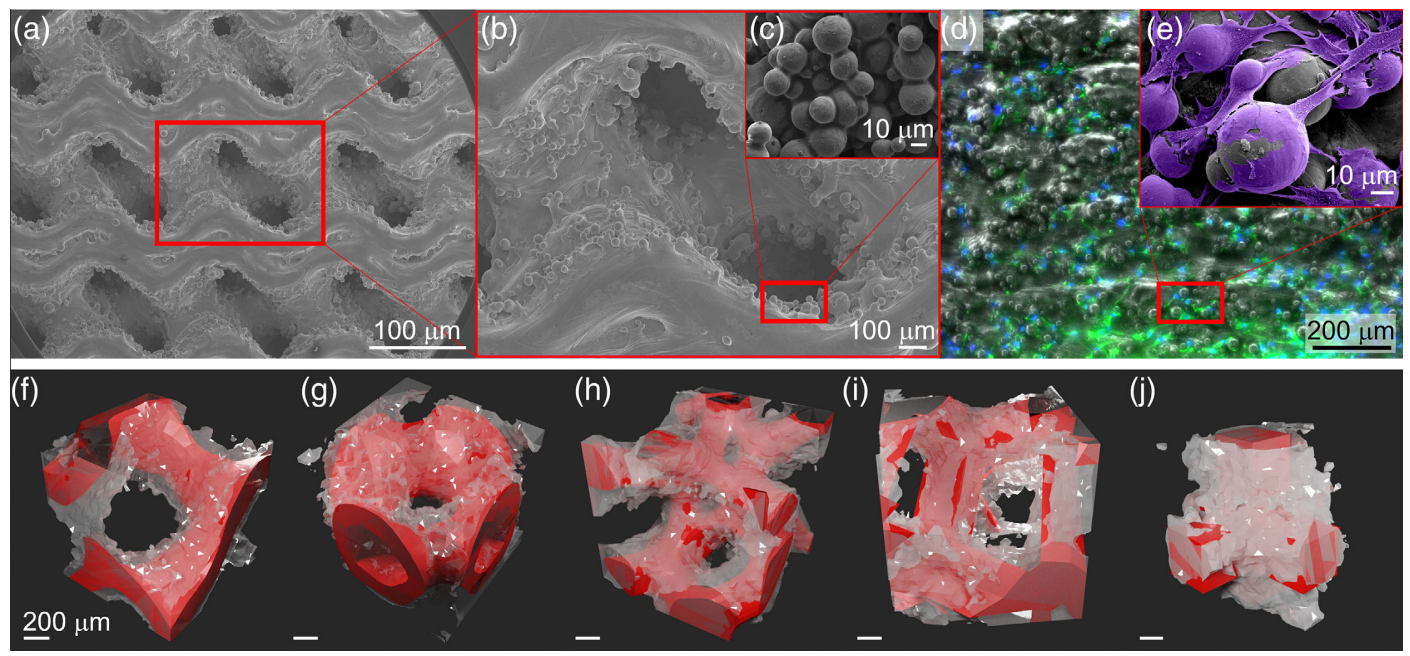

Figure 4. a) SEM observation on a TPMS-gyroid scaffold (middle plane), b,c) zoomed-in details of the previous, d) MC3TE3-C1 preosteoblasts deposited on the "secondary porosity" of cpTi produced by SLM using the laser parameters in this study, and e) zoomed-in details of the previous. After $24 \mathrm{~h}$, the preosteoblasts present extended pseudopods that attach well to the substrate and spread over large areas. The preosteoblasts were stained to display their nucleus (blue) and membrane (green) under fluorescent light (in d) and in purple to distinguish them from the partly sintered powder (in e). Discrepancies study: superposition of printed, $\mu \mathrm{CT}$ scan and reconstructed (grey) unit cells over CAD designs (red) to show discrepancies in the as-manufactured scaffolds due to the presence of "secondary roughness" (i.e., partly melted cpTi particles) in the f) TPMS-gyroid, g) TPMS-IWP, h) T-smooth, i) T-sharp, and j) cubic lattice.

and submicrometer levels has been reported to have a positive effect on bone implant fixation ability. ${ }^{[5]}$

\subsection{Compression Testing}

The scaffolds were subjected to compression testing and the results are shown in Table 1 . Stiffness $\left(E_{\mathrm{p}}\right)$ is similar in all porous scaffolds, with the cubic lattice situated on the upper boundary and the T-sharp on the bottom boundary, including standard deviation (SD) values. This is expected as the spread of apparent actual densities (or porosities) is narrow, and it has been well documented that density is the major driver to changes in stiffness, ${ }^{[7,26]}$ despite differing pore topology. For instance, the compressive properties exhibited by these TPMS-gyroid and -IWP are very similar, and this is in agreement with the comparative results obtained on these topologies albeit for a different raw material. ${ }^{[26 \mathrm{~d}]}$ Yield strength of the porous scaffolds $\left(\sigma_{\mathrm{p}}\right)$ is also similar in all cases and there is even less variation from design to design when considering SD. It is also the case that relative strength is strongly influenced by relative densities. ${ }^{[26 \mathrm{~d}, 27]}$ The strain at yield $\left(\varepsilon_{\mathrm{p}}\right)$ also follows a narrow range, with the 


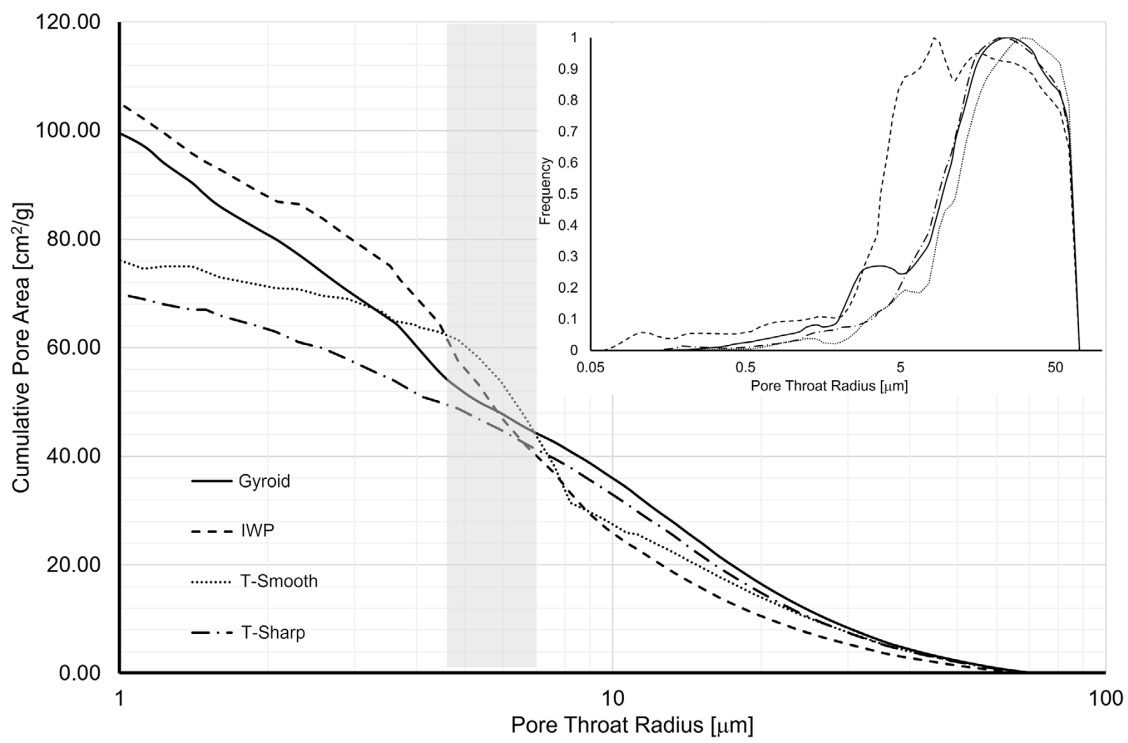

Figure 5. Specific cumulative pore area for the TPMS and trabecular-like scaffolds as a function of pore throat radius and (inset) corresponding pore throat radius distributions. Data collected by mercury porosimetry.

T-sharp setting the upper boundary and therefore appearing to be the most compliant of the set, and the cubic lattice being on the lower boundary, being the least compliant. The stiffness, yield strength, and strain values appear aligned to others reported in literature, though caution must be exercised when comparing different materials (e.g., Ti6Al4 $\mathrm{V}^{[26 \mathrm{~b}]}$ ), actual porosities (e.g., see other studies $\left.{ }^{[7 \mathrm{c}, 16 \mathrm{a}]}\right)$, and strut size or sheet thickness. ${ }^{[12]}$ The trabecular designs' (T-smooth and T-sharp) stiffness and yield strength results aligned very well to the trabecular bone's exponential relationship reported in the study by Ghouse et al., ${ }^{[28]}$ confirming mechano-biomimetic properties. The T-sharp displayed a lower compressive stiffness and yield strength than T-smooth. It is hypothesized that the larger levels of irregularity in the structure realized by the deposition of sintered particles in the sharp bends (Figure 4i), described as crack initiation sites, could be behind this effect, because a similar effect has been reported in highly irregular trabecular-like topologies. ${ }^{[16 a]}$ Observations made from DIC videos report a homogeneous distribution of load across the $X Y$ plane at yield points for the TPMS designs (Figure $6 a, b)$, as expected from other reports: sheet-based gyroids have been reported to transfer uniform load through helical substructures along the vertical axis ${ }^{[29]}$ and IWP deforms collectively. ${ }^{[26 \mathrm{~d}]}$ The trabecular T-smooth and T-sharp designs displayed a similar deformation pattern (Figure 6c,d) with the T-sharp progressing through compression at a faster pace compared to T-Smooth. At yield point, there is still little
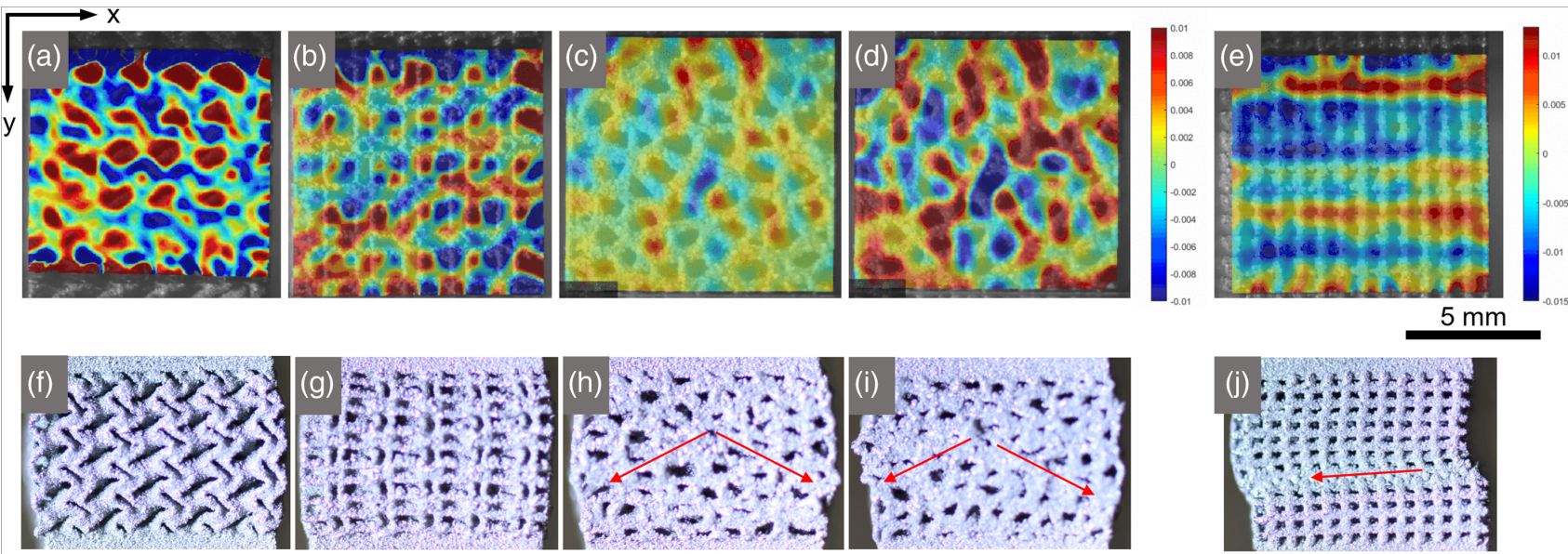

$5 \mathrm{~mm}$

Figure 6. Strain maps (expressed in $\mathrm{mm} / \mathrm{mm}$ ) for the a) gyroid, b) IWP, c) T-smooth, d) T-sharp, and e) cubic lattice on the XY plane at yield points; images of the scaffolds after full compressive deformation: f) gyroid, g) IWP, and after collapse h) T-smooth, i) T-sharp, and j) cubic lattice. Arrows depict orientation and direction of the shear planes. 
evidence of shear planes and the strain map only displays a few compression "cold spots" (in blue). On the contrary, the cubic lattice (Figure 6e) displays an asymmetric compression along the $X Y$ plane. The compressed pores acted as localized stress concentration areas and formed a shear band above which there were examples of buckling and bending. This is attributed to printing imperfections, that the cubic lattice is prone to (Figure 3e), which can cause failure mode transitions. ${ }^{[30]}$ Figure $6 f-j$ shows the final state of the samples after ultimate compressive stress, or collapse, whichever is reached first, and informs about their failure modes-very different because these are highly dependent on unit cell topology. ${ }^{[26 \mathrm{~d}]}$ TPMS-gyroid and -IWP presented barreling, in agreement with other studies ${ }^{[12]}$ and exacerbated by the high friction against the platen due to the sandwich structure, and showed an overall shrinkage of the unit cells, without preferred orientation for deformation and in the absence of shear band formation. This stretching-dominated behavior result agrees with other studies reported in literature for gyroid ${ }^{[26 \mathrm{~b}]}$ and IWP ${ }^{[30 \mathrm{~b}]}$ structures. The T-smooth and -sharp presented a double-shear plane at $45^{\circ}$ upon collapse, forming an inverted "V." The cubic lattice configuration displayed a catastrophic collapse of the middle layers after the deformation strain continued beyond yield point. Those layers collapsed, provoking fracture of the sample (Figure 6j). This failure mechanism was reported in other lattice structures, as it has been demonstrated that although the compressive modulus is larger in the cubic lattice than in, for example, TPMS - gyroid, its shear modulus is the lowest. ${ }^{[6]}$ The results from this study agree with that statement. For trabecular-like topologies, the formation of shear bands has been described in the failure mode of strut-based lattice structures. ${ }^{[30 \mathrm{~b}, 31]}$

\subsection{Biological Results from the In Vitro Studies}

A retention study (i.e., cell attachment) was conducted at $24 \mathrm{~h}$ after seeding to assess any differences in seeding efficiency among the different scaffolds structure (Figure 7a). The results show that at the early stage of attachment onto the scaffolds, the T-sharp presented a slight advantage when compared with the other structures, albeit statistical significance is only with the cubic lattice. The cubic lattice topology was the lowest of the entire set and displayed cellular attrition, with cells percolating fully through the open, thorough-hole structure, offering less resistance to cell suspension and with little oblique surfaces for the preosteoblasts to attach to. This phenomenon has been reported in other cubic lattices ${ }^{[32]}$ and open-strut foam ${ }^{[25 a]}$ configurations. Permeability is therefore an important parameter at the very early stages of the in vitro study. The cubic lattice topology has been reported to have higher permeability than TPMS structures, ${ }^{[6]}$ and when comparing gyroid with IWP, the former has higher permeability, ${ }^{[12]}$ which can explain its lower retention with respect to IWP. The T-sharp scaffolds presented a large amount of sintered and partly melted particles, especially in the corners and joints (Figure 4i), and this can explain why its cell retention is larger, as it has been reported that osteoblasts are better retained onto secondary roughness surfaces. ${ }^{[33]}$

Metabolic activity monitored over 13 days gave an indication of proliferation within the scaffolds (Figure 7b). Preosteoblasts proliferated in all the scaffolds, indicating no cytotoxicity in the environment for them. Cell numbers on the T-sharp scaffolds were higher for the first 7 days, and although there are no statistically significant differences in the results except for day 1 , this (a)

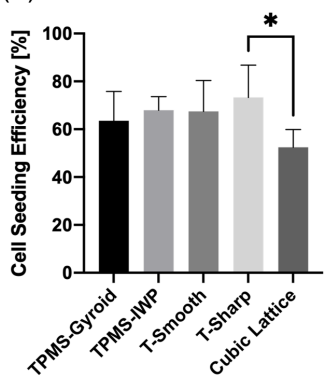

(d)

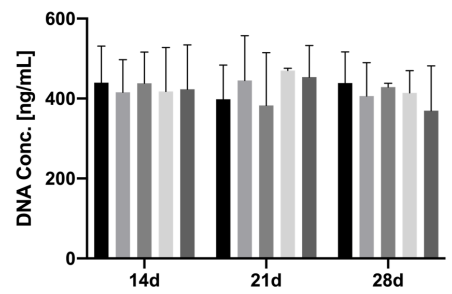

(b)

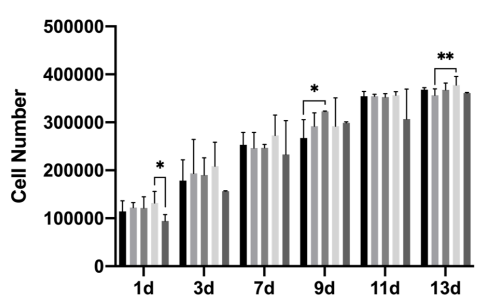

(e)

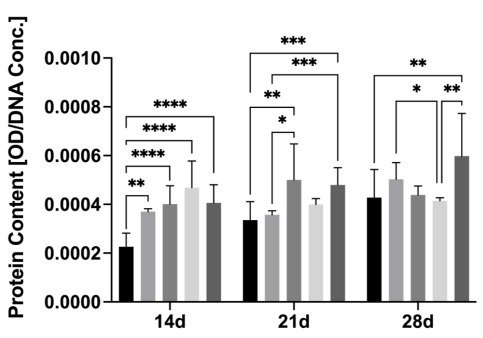

(c)

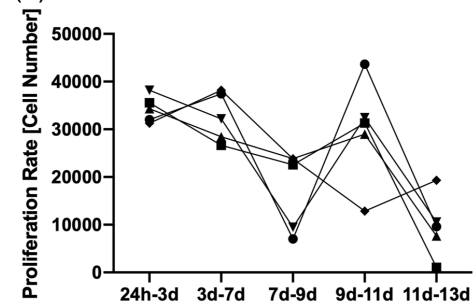

(f)

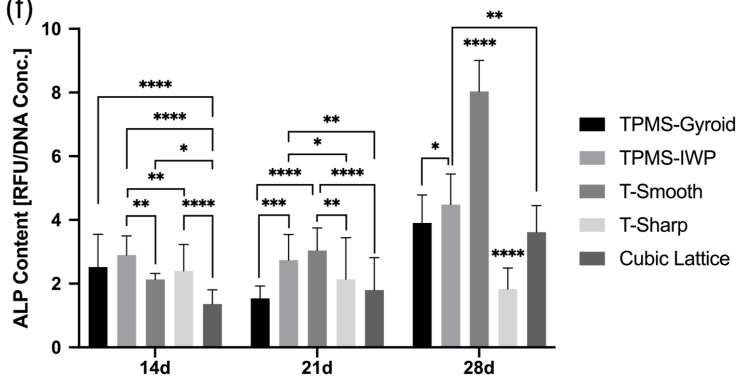

Figure 7. a) Comparative of MC3T3-E1 seeding efficiency at $24 \mathrm{~h}$ indicating cell attachment among different scaffolds, b,c) proliferation study over 13 days reported as absolute cell numbers and rate, respectively, $d-f$ ) long-term differentiation study reporting on (d) DNA content (a surrogate of cell number), (e) protein content normalized by DNA, to indicate individual cell volumes, and ( $f$ ) ALP levels as differentiation markers normalized by DNA content. $(n=4)$ Statistical significance: $* p<0.05, * * p<0.01$, $* * * p<0.001$, and $* * * * p<0.0001$. Lines were used to draw comparisons between topologies, whereas the absence of a line for a marker indicates that significance was universal to all other topologies at that time point. No marker indicates a lack of statistical significance. 
performance could be attributed to the shorter radius of curvature, which makes it more concave, in the strut joints in this structure, subjected to concavity-driven growth acceleration. ${ }^{[34]}$ However, after day 9, except for cubic lattice, cell growth was arrested and phenotype switched, as expected in this cell line. ${ }^{[35]}$ It is by days 11-13 (Figure 7c) that cubic lattice achieved that, and this delay is assumed to be due to the higher attrition and lower cell number in the structure from the outset and therefore a longer time required to reach cell cycle exit conditions. Subsequent increases in cell number are indicative of an increased metabolic activity of senescent maturing cells accommodating higher metabolic activity derived from extracellular matrix (ECM) production.

The differentiation study commenced at day 14 in the cell-laden scaffolds, having confirmed the preosteoblastic phenotypical switch on all scaffolds by that time point. The study was conducted over 28 days to recapitulate a more physiologically relevant in vitro model that results in more translation-ready preclinical data. The DNA content (Figure 7d), indicative of cell number, does not show any significant difference, and the data display large variability. This is indicative of a cellular nonproliferative stage, as expected beyond day 14 . By day 28 , DNA values are equal or lower than that in previous time points, indicating cell number decay due to suspected apoptosis. Protein content values are indicative of cell volume ${ }^{[36]}$ and, when normalized by DNA content, of cellular population morphology (Figure 7e). During long-term culture periods, osteoblasts can go through periods of hypertrophy (i.e., an increase in protein concentration without cellular proliferation) or hyperplasia (i.e., a decrease in matrix protein production). In this study, the volume of osteoblasts cultured in all scaffolds, except in T-sharp, was positively correlated with the study duration, indicating production of matrix proteins. Hypertrophy of osteoblasts in cubic lattice by day 28, with statistical differences, could be explained by the mechanical stimuli experienced by the osteoblasts spanning across corners, which have been reported to promote volume augmentation ${ }^{[32]}$ and commitment toward osteogenesis. On the contrary, hyperplasia observed in the osteoblasts incubated in T-smooth could be a response to decreased metabolic demand due to the completion of a specialized function, such as differentiation. This hypothesis was validated by further investigations of ALP expression.

ALP levels, normalized by DNA content to indicate cellular population maturity (Figure 7f), from osteoblasts cultured on TPMS scaffolds, show that TPMS-IWP upregulated this maturation marker to a larger extent than TPMS-gyroid, with significant differences except for day 14. The ALP levels in T-smooth structures appear upregulated with significant differences, except for day 14 (without statistical significance), compared to T-sharp. This effect is attributed to larger pore sizes, larger surface areas, and larger cumulative pore areas in TPMS-IWP and T-smooth with respect to their counterparts. Large pore sizes are reported to accelerate osteoblast maturation, ${ }^{[15,37]}$ although only surface area was measured in the study by Torres-Sanchez et al. ${ }^{[15]}$ Levels of ALP on the osteoblasts differentiating in cubic lattices were downregulated with respect to the other scaffolds at all time points. These results reached statistical significance in all cases except for day 21 with the TPMS-gyroid and T-sharp and day 28 with the
TPMS-gyroid, where statistical significance is not reached. A smaller pore size could be the reason for this result, and although ALP levels increased throughout the long-term study, indicating osteoblastogenesis, this design appears to be less conducive to maturation than the rest. A preference for irregularity in strutbased scaffolds by human osteosarcoma that showed upregulated ALP levels has been reported. ${ }^{[16 a]}$ However, similar data have not been found for MC3T3-E1. With regard to trabecular-like designs, ALP levels on the T-sharp scaffolds appear stagnant from day 14 to 28 , also indicating that this structure is the least conducive to osteoblastogenesis, and hindered at day 28 with significant statistical differences when compared with all the other structures. This means that osteoblasts incubated in this structure were still in early stages of differentiation as ALP activity is an early marker of osteoblast differentiation that peaks just before matrix mineralization begins. Pore size and strut thickness in T-sharp scaffolds are within the range of the values measured in the other structures. However, a very small cumulative pore area in the T-sharp could be a cause. On the contrary, by day 28, the ALP levels produced by osteoblasts incubated in the Tsmooth scaffolds appeared markedly upregulated with respect to all the other scaffolds with statistical significance. Incidentally, this structure displays the largest cumulative pore area at the scale of pseudopodia size (the shaded area) in Figure 5 and the wider throat radius (Figure 5, inset). There exists a positive correlation between cumulative pore area value (i.e., microporosity) and extent of differentiation by day 28 . Therefore, it can be concluded that this is an important design parameter for implantation scaffolds or 3D networks in tissue engineering. As osteoblasts are known to trigger their differentiation and secretion of ECM by physical cues (namely cell-to-cell direct contact via pseudo- and filopodia interactions ${ }^{[25 a]}$ ) and proximity to other cells, it is hypothesized that T-sharp's higher concavity at the strut joints and corners, which shortens the strutto-strut span and therefore is less demanding for pseudo- and filopodia stretching over gaps, may have inhibited differentiation extent when compared with the ongoing maturation of the osteoblasts in other scaffold designs. The preference of osteoblasts for trabecular-like structures to the detriment of TPMS configurations contributes to the argument that TPMS topologies might not after all be convenient to replicate biological structures as it has been confirmed that trabecular bone does not approximate a minimal surface but exhibits a more intricate curvature landscape. ${ }^{[38]}$

\section{Conclusion}

Efforts to compensate for discrepancies between the as-designed and as-manufactured SLM cpTi scaffolds were made to control the actual porosities and surface areas per unit volume, important drivers in preosteoblastic behavior. As these fell within a tight range, topology comparisons independent from them were possible. As a result, the differences in compression between the TPMS structures were small due to similar apparent porosities, confirming that porosity is a dominant factor over topology design. Both TPMS scaffolds displayed uniform stretchingdominated deformation and similar failure mode. In contrast, the trabecular topologies were more affected by printing 
imperfections, that is, partly sintered particles in corners and joints in the sharper designs, but yielded a stiffer and stronger behavior than TPMS when the topology was smoothened. Both their failure modes displayed double-shear bands, typical for strut-based lattices.

From a bioengineering viewpoint, the sheet TPMS and the strut-based lattices sustained cellular attachment with good retention and were prone to osteoblast proliferation and differentiation. The TPMS-IWP performed better than its TPMSgyroid counterpart, attributed to a larger surface area per unit volume and a higher cumulative pore size (microporosity). This correlation was not found in trabecular-like topologies. Despite a lower surface area per unit volume and lower cumulative pore size, the T-sharp structure promoted a faster proliferation rate, explained by the concavity-driven effect in cellular growth. However, maturation was hindered. It was the T-smooth topology, with a larger surface-to-volume ratio, more accessible pore throats, and larger cumulative pore area at sizes, that matches osteoblast protrusion geometry, which allowed further extension of osteoblastogenesis over the long-term differentiation study.

The results suggest that once macroporosity and pore size values satisfy the minimum requirements of mass transport and mechanical integrity, the most advantageous condition for osteoblastic maturation is offered by a trabecular-like disordered topology with a smoothened finish. The intrinsic roughness generated in the SLM manufacturing process that produced microporosity on the scaffold struts and walls, which particularly favored the T-smooth topology, should also not be disregarded but purposely considered as a design feature as it is believed to have been an important contribution to the larger maturation extent obtained in those scaffolds. Both macro and microporosity, known to be important for cell response and fate, should be included in the design of successful medical implants.

\section{Supporting Information}

Supporting Information is available from the Wiley Online Library or from the author.

\section{Acknowledgements}

The authors gratefully acknowledge EPSRC (grant nos. EP/L014998/1, $\mathrm{EP} / \mathrm{P} 027482 / 1$, and EP/V007335/1) for financial support. Dr J. Wang produced the SMEs for Figure $4 \mathrm{a}-\mathrm{c}$ at the Loughborough Materials Characterisation Centre. Dr E. Demirci generated the $\mu \mathrm{CT}$ scans.

\section{Conflict of Interest}

The authors declare no conflict of interest.

\section{Data Availability Statement}

The data that support the findings of this study are available from the corresponding author upon reasonable request.

\section{Keywords}

commercially pure titanium, gyroids, IWP, osteoblasts, selective laser melting, trabecular, Voronoi tessellations

Received: April 30, 2021

Revised: August 11, 2021

Published online: October 1, 2021

[1] a) G. Bouet, D. Marchat, M. Cruel, L. Malaval, L. Vico, Tissue Eng. Part B, Rev. 2015, 21, 133; b) S. Payr, E. Rosado-Balmayor, T. Tiefenboeck, T. Schuseil, M. Unger, C. Seeliger, M. van Griensven, J. Orthopaedic Surgery Res. 2021, 16, 13.

[2] C. Torres-Sanchez, M. Norrito, J. Wang, H. Bell, L. Zani, P. P. Conway, Surf. Coat. Technol. 2020, 403, 126439.

[3] G. Ryan, A. Pandit, D. P. Apatsidis, Biomaterials 2006, 27, 2651.

[4] V. Karageorgiou, D. Kaplan, Biomaterials 2005, 26, 5474.

[5] F. A. Shah, P. Thomsen, A. Palmquist, Acta Biomater. 2019, 84, 1.

[6] Y. Lu, L. Cheng, Z. Yang, J. Li, H. Zhu, PLOS ONE 2020, 15, 0238471.

[7] a) D. Melancon, Z. S. Bagheri, R. B. Johnston, L. Liu, M. Tanzer, D. Pasini, Acta Biomater. 2017, 63, 350; b) M. Dumas, P. Terriault, V. Brailovski, Mater. Des. 2017, 121, 383; c) D. Barba, E. Alabort, R. C. Reed, Acta Biomater. 2019, 97, 637.

[8] a) S. C. Kapfer, S. T. Hyde, K. Mecke, C. H. Arns, G. E. Schröder-Turk, Biomaterials 2011, 32, 6875; b) O. Al-Ketan, R. Rezgui, R. Rowshan, H. Du, N. X. Fang, R. K. Abu Al-Rub, Adv. Eng. Mater. 2018, 20, 1800029.

[9] H. Chen, Q. Han, C. Wang, Y. Liu, B. Chen, J. Wang, Front. Bioeng. Biotechnol. 2020, 8, 609.

[10] N. Taniguchi, S. Fujibayashi, M. Takemoto, K. Sasaki, B. Otsuki, T. Nakamura, T. Matsushita, T. Kokubo, S. Matsuda, Mater. Sci. Eng.: C 2016, 59, 690.

[11] P. Ouyang, H. Dong, X. He, X. Cai, Y. Wang, J. Li, H. Li, Z. Jin, Mater. Des. 2019, 183, 108151.

[12] F. S. L. Bobbert, K. Lietaert, A. A. Eftekhari, B. Pouran, S. M. Ahmadi, H. Weinans, A. A. Zadpoor, Acta Biomater. 2017, 53, 572.

[13] Y. Du, H. Liang, D. Xie, N. Mao, J. Zhao, Z. Tian, C. Wang, L. Shen, Mater. Chem. Phys. 2020, 239, 121968.

[14] J. Schindelin, I. Arganda-Carreras, E. Frise, V. Kaynig, M. Longair, T. Pietzsch, S. Preibisch, C. Rueden, S. Saalfeld, B. Schmid, J.-Y. Tinevez, D. J. White, V. Hartenstein, K. Eliceiri, P. Tomancak, A. Cardona, Nat. Methods 2012, 9, 676.

[15] C. Torres-Sanchez, M. Norrito, F. R. Almushref, P. P. Conway, Mater. Sci. Eng.: C 2021, 124, 112026.

[16] a) H. Liang, Y. Yang, D. Xie, L. Li, N. Mao, C. Wang, Z. Tian, Q. Jiang, L. Shen, J. Mater. Sci. Technol. 2019, 35, 1284; b) F. Bartolomeu, N. Dourado, F. Pereira, N. Alves, G. Miranda, F. S. Silva, Mater. Sci. Eng.: C 2020, 107, 110342.

[17] D. Mahmoud, M. A. Elbestawi, Int. J. Adv. Manuf. Technol. 2019, 100, 2915.

[18] Z. S. Bagheri, D. Melancon, L. Liu, R. B. Johnston, D. Pasini, J. Mech. Behav. Biomed. Mater. 2017, 70, 17.

[19] Y. Lu, Z. Cui, L. Cheng, J. Li, Z. Yang, H. Zhu, C. Wu, J. Mech. Behav. Biomed. Mater. 2020, 112, 104080.

[20] D. Greitemeier, C. Dalle Donne, F. Syassen, J. Eufinger, T. Melz, Mater. Sci. Technol. 2016, 32, 629.

[21] H. Salem, L. N. Carter, M. M. Attallah, H. G. Salem, Mater. Sci. Eng.: A 2019, 767, 138387.

[22] a) K. F. Walker, Q. Liu, M. Brandt, Int. J. Fatigue 2017, 104, 302 b) I. Koutiri, E. Pessard, P. Peyre, O. Amlou, T. De Terris, J. Mater. Process. Technol. 2018, 255, 536. 
[23] a) K. Anselme, P. Linez, M. Bigerelle, D. Le Maguer, A. Le Maguer, P. Hardouin, H. F. Hildebrand, A. lost, J. M. Leroy, Biomaterials 2000 , 21, 1567; b) K. M. Hotchkiss, G. B. Reddy, S. L. Hyzy, Z. Schwartz, B. D. Boyan, R. Olivares-Navarrete, Acta Biomater. 2016, 31, 425.

[24] W. Xue, B. V. Krishna, A. Bandyopadhyay, S. Bose, Acta Biomater. 2007, 3, 1007.

[25] a) K. C. Nune, R. D. K. Misra, S. M. Gaytan, L. E. Murr, J. Biomed. Mater. Res. Part A 2015, 103, 1677; b) C. Wang, X.-d. Xie X. Huang, Z.-h. Liang, C.-r. Zhou, Colloids Surf. B: Biointerfaces 2015, 132, 1.

[26] a) D. W. Abueidda, R. K. Abu Al-Rub, A. S. Dalaq, D.-W. Lee, K. A. Khan, I. Jasiuk, Mech. Mater. 2016, 95, 102; b) C. N. Kelly, J. Francovich, S. Julmi, D. Safranski, R. E. Guldberg, H. J. Maier, K. Gall, Acta Biomater. 2019, 94, 610; c) C. Torres-Sanchez, J. McLaughlin, R. Bonallo, J. Mater. Eng. Perform. 2018, 27, 2899; d) O. Al-Ketan, R. Rowshan, R. K. Abu Al-Rub, Addit. Manuf. 2018, 19, 167.

[27] C. Yan, L. Hao, A. Hussein, P. Young, J. Mech. Behav. Biomed. Mater. 2015, 51, 61 .

[28] S. Ghouse, N. Reznikov, O. R. Boughton, S. Babu, K. C. G. Ng, G. Blunn, J. P. Cobb, M. M. Stevens, J. R. T. Jeffers, Appl. Mater. Today 2019, 15, 377.
[29] D. Downing, A. Jones, M. Brandt, M. Leary, Mater. Des. 2021, 197, 109096.

[30] a) L. Liu, P. Kamm, F. García-Moreno, J. Banhart, D. Pasini, J. Mech. Phys. Solids 2017, 107, 160; b) O. Al-Ketan, R. K. Abu Al-Rub, R. Rowshan, J. Mater. Res. 2018, 33, 343.

[31] M. Smith, Z. Guan, W. J. Cantwell, Int. J. Mech. Sci. 2013, 67, 28.

[32] S. Van Bael, Y. C. Chai, S. Truscello, M. Moesen, G. Kerckhofs, H. Van Oosterwyck, J. P. Kruth, J. Schrooten, Acta Biomater. 2012, 8, 2824.

[33] B. Wysocki, J. Idaszek, J. Buhagiar, K. Szlązak, T. Brynk, K. J. Kurzydłowski, W. Święszkowski, Mater. Sci. Eng.: C 2019, 95, 428.

[34] M. Rumpler, A. Woesz, J. W. C. Dunlop, J. T. v. Dongen, P. Fratzl, J. R. Soc. Interface 2008, 5, 1173.

[35] L. D. Quarles, D. A. Yohay, L. W. Lever, R. Caton, R. J. Wenstrup, J. Bone Min. Res. 1992, 7, 683.

[36] H. A. Crissman, J. A. Steinkamp, J. Cell Biol. 1973, 59, 766.

[37] K. Kapat, P. K. Srivas, A. P. Rameshbabu, P. P. Maity, S. Jana, J. Dutta, P. Majumdar, D. Chakrabarti, S. Dhara, ACS Appl. Mater. Interfaces 2017, 9, 39235.

[38] S. J. P. Callens, D. C. Tourolle né Betts, R. Müller, A. A. Zadpoor, Acta Biom, 2021, 130, 343. 